\title{
Semigroup cohomology and applications
}

\author{
B. V. Novikov (Kharkov, Ukraine)
}

This article is a survey of the author's research. It consists of three sections concerned three kinds of cohomologies of semigroups. Section 1 considers 'classic' cohomology as it was introduced by Eilenberg and MacLane. Here the attention is concentrated mainly on semigroups having cohomological dimension 1. In Section 2 a generalization of the Eilenberg-MacLane cohomology is introduced, the so-called 0-cohomology, which appears in applied topics (projective representations of semigroups, Brauer monoids). At last Section 3 is devoted to further generalizing: partial cohomology defined and discussed in it are used then for calculation of the classic cohomology for some semigroups.

I am indebted to Prof. K. Roggenkamp and Prof. M. Ştefanescu for the support of my participation in the Workshop.

\section{EM-cohomology}

In this section we deal with the Eilenberg-MacLane cohomology of semigroups [3] (shortly EM-cohomology). Its definition is the same as for groups:

$$
H^{n}(S, A)=\operatorname{Ext}_{\mathbf{Z} S}^{n}(\mathbf{Z}, A) .
$$

Here $S$ is a semigroup, $A$ a left $S$-module (i. e. a left module over the integral semigroup ring $\mathbf{Z} S$ ), $\mathbf{Z}$ is considered as a trivial $S$-module (i. e. $x a=a$ for all $x \in S, a \in \mathbf{Z}) ; H^{n}(S, A)$ is called $a n^{\text {th }}$ cohomology group of $S$ (with the coefficient module $A)$.

Another definition (equivalent to preceding one) of $H^{n}(S, A)$ is following. Denote by $C^{n}(S, A)$ the group of all maps $f: \underbrace{S \times \ldots \times S}_{n \text { times }} \rightarrow A$ (a group of 
$n$-cochains); a coboundary homomorphism $\partial^{n}: C^{n}(S, A) \rightarrow C^{n+1}(S, A)$ is given by the formula

$$
\begin{aligned}
& \partial^{n} f\left(x_{1}, \ldots, x_{n+1}\right)=x_{1} f\left(x_{2}, \ldots, x_{n+1}\right) \\
& +\sum_{i=1}^{n}(-1)^{i} f\left(x_{1}, \ldots, x_{i} x_{i+1}, \ldots, x_{n+1}\right)+(-1)^{n+1} f\left(x_{1}, \ldots, x_{n}\right)
\end{aligned}
$$

Then $\partial^{n} \partial^{n-1}=0$, i.e.

$$
\begin{gathered}
\operatorname{Im} \partial^{n-1}=B^{n}(S, A) \text { (a group of coboundaries) } \\
\subseteq \operatorname{Ker} \partial^{n}=Z^{n}(S, A) \text { (a group of cocycles) }
\end{gathered}
$$

and cohomology groups are defined as $H^{n}(S, A)=Z^{n}(S, A) / B^{n}(S, A)$.

It is worth noting two simple properties of the semigroup cohomology [1] which are useful below.

(1) Adjoin to a given semigroup $S$ an extra element 1 and extend the operation (multiplication) of $S$ to $S^{1}=S \cup\{1\}$ by

$$
\forall s \in S \quad s \cdot 1=1 \cdot s=s, \quad 1 \cdot 1=1 .
$$

Then $S^{1}$ becomes a monoid (i. e. a semigroup with an identity element) and is called a semigroup with an adjoint identity. Every $S$-module turns naturally into a (unitary) $S^{1}$-module and we have

$$
H^{n}\left(S^{1}, A\right) \cong H^{n}(S, A), \quad n \geq 0 .
$$

(2) If $S$ possesses a zero element 0 then $H^{n}(S, A)=0$ for each $S$-module $A$ and for all $n \geq 1$. In particular, for any semigroup $S$ we can define the semigroup $S^{0}$ with an adjoint zero (analogously to $S^{1}$ ); then we have $H^{n}\left(S^{0}, A\right)=0(n \geq 1)$.

The semigroup EM-cohomology has not so wide applications as the cohomology of groups. Nevertheless it is interesting for homologists at least as a model for testing homological methods. The problem of describing semigroups having cohomological dimension 1 is such an example. This problem has its own story.

Cohomological dimension (c. d.) of a semigroup $S$ is the maximal integer $n$ such that $H^{n}(S, A) \neq 0$ for some $S$-module $A$. There are many reasons to study algebraic objects of c.d.1 (see, e.g., [4]). 
It is an easy exercise to prove that both a free group and a free semigroup (or a free monoid) have c.d. 1 [3]. For groups the converse is true - this is the well-known Stallings-Swan theorem [2]. So a group has cohomological dimension one if and only if it is free.

Now, what about semigroups?

First, as we have mentioned above, every semigroup with 0 has c.d. $\leq 1$. This is the main reason why we have to confine ourselves to considering cancellative semigroups only.

Second, a free group is not free as a semigroup. So even for cancellative semigroups the Stallings-Swan theorem doesn't hold.

B. Mitchell [13] has shown that a so-called partially free monoid (the free product of a free group and a free monoid) has c.d.1. He has supposed that if c. d. $S=1$ for $S$ cancellative then $S$ is partially free.

In [16] I have built the first counter-example for Mitchell conjecture:

$$
S=\langle a, b, c, d \mid a b=c d\rangle
$$

and in [17] I have formulated a 'weakened Mitchell conjecture'. It turned out true:

Theorem 1 [22] Every cancellative semigroup of c.d.1 can be embedded into a free group.

In the proof of this theorem the passage from the homological language to the semigroup one is realized by the following lemma (which may be helpful not only for semigroups).

Let $A$ be a left module over an arbitrary ring $R$,

$$
\stackrel{\stackrel{d_{2}}{\longrightarrow}}{\longrightarrow} P_{2} \stackrel{d_{1}}{\longrightarrow} P_{1} \stackrel{d_{0}}{\longrightarrow} P_{0} \longrightarrow 0
$$

its projective resolution. Evidently, $d_{n}$ may be considered as a $(n+1)$ dimensional cocycle with values in the $R$-module $\operatorname{Im} d_{n}$.

Lemma 2 The cocycle $d_{n} \in Z^{n+1}\left(A, \operatorname{Im} d_{n}\right)$ is a coboundary iff the projective dimension of $A$ is not greater than $n$.

Applying Lemma 2 to a bar-resolution of the $S$-module $\mathbf{Z}$ we obtain the next property of a cancellative semigroup $S$ with c.d. 1 . Consider a graph 
with the elements of $S$ as vertices and with the pairs $(a, b) \in S \times S$ such that $a S \cap b S \neq \emptyset$ as edges. Then every circuit of this graph is triangulable.

This property allows to prove that $S$ can be embedded into a group (the latter turns out free by the Stallings-Swan theorem). Note that the converse assertion is certainly not true: there is a subsemigroup of a free semigroup, which has c.d.1, while c.d. of its anti-isomorphic is equal to 2 (see the examples below). A nice answer is only obtained in the commutative case [19]: the c.d. of a commutative cancellative semigroup is equal to 1 if and only if this semigroup can be embedded into $\mathbf{Z}$.

So a new problem arises: to describe subsemigroups of a free group having c.d.1. This question seems rather difficult even if we restrict ourselves to subsemigroups of a free semigroup. The following results are taken out of [24].

Let $S$ be a subsemigroup of a free semigroup $F$. Further development of the proof of Theorem 1 gives us the next assertion:

Theorem 3 Let c.d. $S=1$ and $a S \cap S \neq \emptyset \neq S a \cap S$ for some $a \in F \backslash S$. There exists such $x \in a S \cap S$ that $a S \cap S \subset x F^{1}$.

This theorem allows to build a lot of subsemigroups of $F$ having c.d. $>1$.

Example 1 Let $a, p, q, r$ are different elements of $F$ such that:

1) $\min (|a|,|p|,|q|,|r|)=|a|(|a|$ denotes the length of the word $a)$,

2) $p$ and $q$ begin with different letters.

Then the subsemigroup $S=\langle p, q, r, a p, a q, r a\rangle$ has c.d. $>1$.

From Theorem 3 a solution of the proposed problem for left ideals is obtained:

Proposition 4 A left ideal of a free semigroup has c.d. 1 iff it is free.

Corollary 5 Every proper two-sided ideal of a free semigroup has c.d. $>1$.

For principal right ideals the situation is similar:

Proposition 6 A principal right ideal of a free semigroup has c.d.1 iff it is free. 
However for arbitrary right ideals the analog of Proposition 4 is not true:

Example 2 Let $F=\langle a, b\rangle$ be a free semigroup. Then $R=\{b, a b a\} F^{1}$ is not free but c. d. $R=1$.

By the way, from these results a counter-example to another conjecture follows. Yu. Drozd supposed that for any $S \in F$ either $S$ or the antiisomorphic to $S$ has c.d.1. Consider the principal left ideal $L=F^{1} a b a$ in a free semigroup $F=\langle a, b\rangle$. It is not free since its generators $a b a,(a b)^{2},(a b)^{2} a$, $(a b)^{3}$ obey the relation

$$
(a b)^{2} \cdot(a b)^{2} a=(a b)^{3} \cdot a b a .
$$

By Proposition 4 c.d. $L>1$. Of course its antiisomorphic $R=a b a F^{1}$ is not free too and c.d. $R>1$ by Proposition 6 . Hence the pair $(L, R)$ gives a counter-example to the conjecture.

\section{0-cohomology}

In order to see how 0-cohomology appears let us try to define a projective representation of a semigroup.

Let $K$ be a field, $K^{\times}$its multiplicative group, $n$ a positive integer, $M(n, K)$ the semigroup of all $n \times n$ matrices over $K$. Define an equivalence: for $A, B \in M(n, K)$

$$
A \sim B \Longleftrightarrow \exists \lambda \in K^{\times} A=\lambda B
$$

Then $\sim$ is a congruence on the semigroup $M(n, K)$ and we can consider a factor semigroup $P M(n, K)=M(n, K) / \sim$, 'the projective linear semigroup'.

Like for groups we call a projective representation of a given semigroup $S$ a homomorphism $\Gamma: S \rightarrow P M(n, K)$.

Fix an element in each $\sim$-class. Then $\Gamma$ induces a map $\Gamma^{\prime}: S \rightarrow M(n, K)$. Now we can redefine a projective representation of $S$ : it is a map $\Gamma^{\prime}: S \rightarrow$ $M(n, K)$ such that

1) $\Gamma^{\prime}(x) \Gamma^{\prime}(y)=0 \Longleftrightarrow \Gamma^{\prime}(x y)=0$,

2) $\Gamma^{\prime}(x) \Gamma^{\prime}(y)=\Gamma^{\prime}(x y) \rho(x, y)$, where $\rho: S \times S \rightarrow K^{\times}$is a partial function defined on the subset $\left\{(x, y) \mid \Gamma^{\prime}(x y) \neq 0\right\}$. 
Certainly $\rho$ yields the equation

$$
\rho(x, y) \rho(x y, z)=\rho(x, y z) \rho(y, z)
$$

for $\Gamma^{\prime}(x y z) \neq 0$ and can be used as the corresponding 2-cocycle (like a factor system in Group Theory) excepting its partiality. Therefore we must anew define suitable cohomology as follows.

Let $S$ be an arbitrary semigroup with a zero. An Abelian group $A$ is called a 0-module over $S$, if an action $(S \backslash\{0\}) \times A \rightarrow A$ is defined which satisfies for all $s, t \in S \backslash\{0\}, a, b \in A$ the following conditions:

$$
\begin{gathered}
s(a+b)=s a+s b, \\
s t \neq 0 \Longrightarrow s(t a)=(s t) a .
\end{gathered}
$$

$A n$-dimensional 0 -cochain is a partial $n$-place map from $S$ to $A$ which is defined for all $n$-tuples $\left(s_{1}, \ldots, s_{n}\right)$, such that $s_{1} \cdot \ldots \cdot s_{n} \neq 0$. A coboundary homomorphism is given like for the usual cohomology by the formula (1). The equality $\partial^{n} \partial^{n-1}=0$ is valid too. We denote

$$
\begin{gathered}
\operatorname{Im} \partial^{n-1}=B_{0}^{n}(S, A) \text { (a group of 0-coboundaries) } \\
\subseteq \operatorname{Ker}^{n}=Z_{0}^{n}(S, A) \text { (a group of 0-cocycles) }
\end{gathered}
$$

and 0 -cohomology groups are defined as $H_{0}^{n}(S, A)=Z_{0}^{n}(S, A) / B_{0}^{n}(S, A)$.

Note that for a semigroup $T^{0}=T \cup\{0\}$ with an adjointed zero

$$
H_{0}^{n}\left(T^{0}, A\right) \cong H^{n}(T, A)
$$

so the 0-cohomology may be considered as a generalization of the EilenbergMacLane cohomology.

Properties of 0-cohomology are not considered here since they follow from the properties of partial cohomologies (see Section 3).

Before returning to the projective representations we need a semigrouptheoretic construction, the so-called semilattice of groups [6].

Let $\Lambda$ be a semilattice (i. e. a partially ordered set in which every two elements $\lambda, \mu$ have the greatest lower bound $\lambda \mu$ ) and let $\left\{G_{\lambda} \mid \lambda \in \Lambda\right\}$ be a family of disjoint groups. For each pair $\lambda, \mu \in \Lambda$ such that $\lambda \geq \mu$, let $\varphi_{\mu}^{\lambda}: G_{\lambda} \rightarrow G_{\mu}$ be a homomorphism. Suppose that 
1) $\varphi_{\lambda}^{\lambda}$ is identical for every $\lambda \in \Lambda$,

2) $\varphi_{\mu}^{\lambda} \varphi_{\nu}^{\mu}=\varphi_{\nu}^{\lambda}$ for all $\lambda \geq \mu \geq \nu$.

Define a multiplication on the set $T=\cup_{\lambda \in \Lambda} G_{\lambda}$ by the rule: if $x \in G_{\lambda}$, $y \in G_{\mu}$

$$
x y=\left(\varphi_{\lambda \mu}^{\lambda} x\right)\left(\varphi_{\lambda \mu}^{\mu} y\right) .
$$

Then $T$ becomes a semigroup which is called a semilattice of groups.

Now return to the projective representations. Recall [8] that if $S$ is a group then one defines an equivalence on the set of the factor systems of $S$ (which corresponds to the equivalence of projective representations); the factor set by this equivalence is a group $\operatorname{Sch}(S, K)$ which is called a Schur multiplicator and describes (in some sense) all projective representations of $S$ over $K$. It is well-known that $\operatorname{Sch}(S, K) \cong H^{2}\left(S, K^{\times}\right)$, where $K^{\times}$is considered as a trivial $S$-module.

What will be for semigroups? In this situation $\operatorname{Sch}(S, K)$ is not a group (more exactly, it becomes an inverse semigroup). Let $\Lambda$ be a semilattice of all two-sided ideals of $S$ (including $\emptyset$ and $S$ ) with respect to the inclusion and the union as a greatest lower bound. Then restriction of 0-cochains induces homomorphisms

$$
\varphi_{J}^{I}: H_{0}^{n}\left(S / I, K^{\times}\right) \longrightarrow H_{0}^{n}\left(S / J, K^{\times}\right)
$$

for ideals $I \subseteq J$ and we have a semilattice of groups $\bigcup_{I \in \Lambda} H_{0}^{n}\left(S / I, K^{\times}\right)$(here for $I=\emptyset$ we set $\left.H_{0}^{n}\left(S / \emptyset, K^{\times}\right)=H^{n}\left(S, K^{\times}\right)\right)$. The next assertion was proved in $[15]$ :

Theorem 7 For every semigroup $S$ and every field $K$

$$
\operatorname{Sch}(S, K) \cong \bigcup_{I \in \Lambda} H_{0}^{2}\left(S / I, K^{\times}\right)
$$

Note that even if $0 \notin S$ we have to use 0 -cohomology for describing of $\operatorname{Sch}(S, K)$.

Another application of the 0-cohomology appears in connection with the Brauer monoid. This notion was introduced by Haile, Larson and Sweedler [9], [10] while they studied the so-called strongly primary algebras (a generalization of central simple ones). I shall not give their original definition which is rather complicated. But it turned out that the Brauer monoid can 
be defined in terms of the 0-cohomology [20]. To do it one must introduce a new notion, a modification of a group.

By a modification $G(*)$ of a group $G$ we mean a semigroup on the set $G^{0}=G \cup\{0\}$ with an operation $*$ such that $x * y$ is equal either to $x y$ or to 0 , while

$$
0 * x=x * 0=0 * 0=0
$$

and the identity of $G$ is the same for the semigroup $G(*)$.

In other words, to obtain a modification, one must erase the contents of some inputs in the multiplication table of $G$ and insert there zeros so that the new operation would be associative.

Note some general properties of modifications. First, a modification of $G$ satisfies the weak cancellation condition: from $x * z=y * z \neq 0$ it follows $x=y$ and analogously for left cancellation. Second, let $U$ be a subgroup of all invertible elements in $G(*)$. Then its complement $I=G(*) \backslash U$ is a two-sided ideal. One can show that if $G$ is finite, $I$ is nilpotent.

Let $S=G(*)$ and $T=G(\star)$ be modifications of $G$. It is clear that $S \cap T=G(\circ)$ is a modification too, where

$$
x \circ y \neq 0 \Longleftrightarrow x * y \neq 0 \neq x \star y \text {. }
$$

We write $S \prec T$ if $x \star y=0$ implies $x * y=0$ for all $x, y \in G$. Obviously, all modifications of $G$ constitute a semilattice $M(G)$ : the greatest lower bound in it is $S \cap T$.

Each $G$-module $A$ can be turned into a 0-module over a modification $S$ in a natural way. Moreover, if $S \prec T$ then each 0 -module over $T$ is transformed into a 0 -module over $S$. Therefore for $S \prec T$ a homomorphism is defined

$$
\varphi_{S}^{T}: H_{0}^{n}(T, A) \longrightarrow H_{0}^{n}(S, A)
$$

and we obtain a semilattice of groups $\bigcup_{S \in M(G)} H_{0}^{n}(S, A)$.

In particular, let $L$ be a finite-dimensional normal extension of a field $K$ with the Galois group $G$. Then $L^{\times}$is a $G$-module. We define a (relative) Brauer monoid as

$$
\operatorname{Br}(G, L)=\bigcup_{S \in M(G)} H_{0}^{2}\left(S, L^{\times}\right)
$$

(the adjective 'relative' will be omitted since in this article relative Brauer monoids are only considered). 
In the case when operation $*$ is defined in such a way that $x * y=x y$ for $x, y \neq 0$, we have

$$
H_{0}^{2}\left(S, L^{\times}\right) \cong H^{2}\left(G, L^{\times}\right),
$$

so the Brauer group is a subgroup of the Brauer monoid.

One can hope that the Brauer monoid will be useful. For example, it is well-known that the Brauer group is trivial for any finite field whereas the Brauer monoid is not trivial for each non-trivial field extension.

The Brauer monoid classifies strongly primary algebras over a field like the Brauer group classifies division algebras.

The use of 0-cohomology allows us to split the study of the Brauer monoid into two problems:

1) describing all modifications of a given finite group,

2) computing 0-cohomology of a modification.

Both of them seem rather difficult, especially the first. Its solution is unknown even for cyclic groups. In [21] some class of modifications of simple cyclic groups is described. It implies that the number of modifications of the group $\mathbf{Z}_{p}$ is $O\left(p^{2}\right)$. All multiplication tables of the modifications $S_{1}, \ldots, S_{15}$ of $\mathbf{Z}_{5}$ (up to automorphisms of the group) are shown in Table 1; for $\mathbf{Z}_{7}$ their number equals 145 .

As to the second problem, the initial step in solving it may consist in eliminating the influence of invertible elements of modifications on the structure of the Brauer monoid. Some results in this direction were obtained in [12], [20].

As above let $G$ be the Galois group of a finite-dimensional extension $L / K$, $S=G(*)$ its modification, $U$ the subgroup of invertible elements of $S, P$ the subfield of all $U$-fixed elements: $P=\{a \in L \mid U a=a\}$. The inclusion $U \hookrightarrow S$ induces a homomorphism

$$
\psi: H_{0}^{2}\left(S, L^{\times}\right) \longrightarrow H^{2}\left(U, L^{\times}\right)
$$

We shall study this homomorphism in the situation when $U$ is a normal subgroup of $S$ (i. e. $x * U=U * x$ for all $x \in S$ ). Then the factor semigroup $S / U$ is well-defined.

Further, if $U \triangleleft S$ then $P^{\times}$is a 0 -module over $S / U$. The inclusion $P^{\times} \hookrightarrow L^{\times}$ and the epimorphism $S \rightarrow S / U$ induce a homomorphism

$$
\chi: H_{0}^{2}\left(S / U, P^{\times}\right) \longrightarrow H_{0}^{2}\left(S, L^{\times}\right)
$$




\begin{tabular}{|c||c|c|c|c|}
\hline$S_{1}$ & $a^{1}$ & $a^{2}$ & $a^{3}$ & $a^{4}$ \\
\hline$a^{1}$ & 0 & 0 & 0 & 0 \\
\hline$a^{2}$ & 0 & $a^{4}$ & 0 & $a^{1}$ \\
\hline$a^{3}$ & 0 & 0 & $a^{1}$ & 0 \\
\hline$a^{4}$ & 0 & $a^{1}$ & 0 & 0 \\
\hline
\end{tabular}

\begin{tabular}{|c|c|c|c|c|}
\hline$S_{2}$ & $a^{1}$ & $a^{2}$ & $a^{3}$ & $a^{4}$ \\
\hline$a^{1}$ & 0 & 0 & 0 & 0 \\
\hline$a^{2}$ & 0 & $a^{4}$ & 0 & 0 \\
\hline$a^{3}$ & 0 & 0 & $a^{1}$ & 0 \\
\hline$a^{4}$ & 0 & 0 & 0 & 0 \\
\hline
\end{tabular}

\begin{tabular}{|c|c|c|c|c|}
\hline$S_{3}$ & $a^{1}$ & $a^{2}$ & $a^{3}$ & $a^{4}$ \\
\hline$a^{1}$ & 0 & 0 & 0 & 0 \\
\hline$a^{2}$ & 0 & 0 & 0 & $a^{1}$ \\
\hline$a^{3}$ & 0 & 0 & $a^{1}$ & $a^{2}$ \\
\hline$a^{4}$ & 0 & $a^{1}$ & $a^{2}$ & $a^{3}$ \\
\hline
\end{tabular}

\begin{tabular}{|c|c|c|c|c|}
\hline$S_{4}$ & $a^{1}$ & $a^{2}$ & $a^{3}$ & $a^{4}$ \\
\hline$a^{1}$ & 0 & 0 & 0 & 0 \\
\hline$a^{2}$ & 0 & 0 & 0 & $a^{1}$ \\
\hline$a^{3}$ & 0 & 0 & $a^{1}$ & 0 \\
\hline$a^{4}$ & 0 & $a^{1}$ & 0 & 0 \\
\hline
\end{tabular}

\begin{tabular}{|c|c|c|c|c|}
\hline$S_{5}$ & $a^{1}$ & $a^{2}$ & $a^{3}$ & $a^{4}$ \\
\hline$a^{1}$ & 0 & 0 & 0 & 0 \\
\hline$a^{2}$ & 0 & 0 & 0 & $a^{1}$ \\
\hline$a^{3}$ & 0 & 0 & $a^{1}$ & 0 \\
\hline$a^{4}$ & 0 & 0 & 0 & 0 \\
\hline
\end{tabular}

\begin{tabular}{|c|c|c|c|c|}
\hline$S_{6}$ & $a^{1}$ & $a^{2}$ & $a^{3}$ & $a^{4}$ \\
\hline$a^{1}$ & 0 & 0 & 0 & 0 \\
\hline$a^{2}$ & 0 & 0 & 0 & 0 \\
\hline$a^{3}$ & 0 & 0 & $a^{1}$ & $a^{2}$ \\
\hline$a^{4}$ & 0 & 0 & $a^{2}$ & 0 \\
\hline
\end{tabular}

\begin{tabular}{|c|c|c|c|c|}
\hline$S_{7}$ & $a^{1}$ & $a^{2}$ & $a^{3}$ & $a^{4}$ \\
\hline$a^{1}$ & 0 & 0 & 0 & 0 \\
\hline$a^{2}$ & 0 & 0 & 0 & 0 \\
\hline$a^{3}$ & 0 & 0 & $a^{1}$ & 0 \\
\hline$a^{4}$ & 0 & $a^{1}$ & 0 & 0 \\
\hline
\end{tabular}

\begin{tabular}{|c|c|c|c|c|}
\hline$S_{8}$ & $a^{1}$ & $a^{2}$ & $a^{3}$ & $a^{4}$ \\
\hline$a^{1}$ & 0 & 0 & 0 & 0 \\
\hline$a^{2}$ & 0 & 0 & 0 & 0 \\
\hline$a^{3}$ & 0 & 0 & $a^{1}$ & 0 \\
\hline$a^{4}$ & 0 & 0 & $a^{2}$ & 0 \\
\hline
\end{tabular}

\begin{tabular}{|c|c|c|c|c|}
\hline$S_{9}$ & $a^{1}$ & $a^{2}$ & $a^{3}$ & $a^{4}$ \\
\hline$a^{1}$ & 0 & 0 & 0 & 0 \\
\hline$a^{2}$ & 0 & 0 & 0 & 0 \\
\hline$a^{3}$ & 0 & 0 & 0 & $a^{2}$ \\
\hline$a^{4}$ & 0 & 0 & $a^{2}$ & 0 \\
\hline
\end{tabular}

\begin{tabular}{|c|c|c|c|c|}
\hline$S_{10}$ & $a^{1}$ & $a^{2}$ & $a^{3}$ & $a^{4}$ \\
\hline$a^{1}$ & 0 & 0 & 0 & 0 \\
\hline$a^{2}$ & 0 & 0 & 0 & 0 \\
\hline$a^{3}$ & 0 & 0 & 0 & $a^{2}$ \\
\hline$a^{4}$ & 0 & 0 & $a^{2}$ & $a^{3}$ \\
\hline
\end{tabular}

\begin{tabular}{|c|c|c|c|c|}
\hline$S_{11}$ & $a^{1}$ & $a^{2}$ & $a^{3}$ & $a^{4}$ \\
\hline$a^{1}$ & 0 & 0 & 0 & 0 \\
\hline$a^{2}$ & 0 & 0 & 0 & 0 \\
\hline$a^{3}$ & 0 & 0 & 0 & 0 \\
\hline$a^{4}$ & 0 & $a^{1}$ & 0 & 0 \\
\hline
\end{tabular}

\begin{tabular}{|c|c|c|c|c|}
\hline$S_{12}$ & $a^{1}$ & $a^{2}$ & $a^{3}$ & $a^{4}$ \\
\hline$a^{1}$ & 0 & 0 & 0 & 0 \\
\hline$a^{2}$ & 0 & 0 & 0 & 0 \\
\hline$a^{3}$ & 0 & 0 & 0 & 0 \\
\hline$a^{4}$ & 0 & $a^{1}$ & 0 & $a^{3}$ \\
\hline
\end{tabular}

\begin{tabular}{|c|c|c|c|c|}
\hline$S_{13}$ & $a^{1}$ & $a^{2}$ & $a^{3}$ & $a^{4}$ \\
\hline$a^{1}$ & 0 & 0 & 0 & 0 \\
\hline$a^{2}$ & 0 & 0 & 0 & 0 \\
\hline$a^{3}$ & 0 & 0 & 0 & 0 \\
\hline$a^{4}$ & 0 & 0 & $a^{2}$ & 0 \\
\hline
\end{tabular}

\begin{tabular}{|c|c|c|c|c|}
\hline$S_{14}$ & $a^{1}$ & $a^{2}$ & $a^{3}$ & $a^{4}$ \\
\hline$a^{1}$ & 0 & 0 & 0 & 0 \\
\hline$a^{2}$ & 0 & 0 & 0 & 0 \\
\hline$a^{3}$ & 0 & 0 & 0 & 0 \\
\hline$a^{4}$ & 0 & 0 & 0 & 0 \\
\hline
\end{tabular}

\begin{tabular}{|c|c|c|c|c|}
\hline$S_{15}$ & $a^{1}$ & $a^{2}$ & $a^{3}$ & $a^{4}$ \\
\hline$a^{1}$ & 0 & 0 & 0 & 0 \\
\hline$a^{2}$ & 0 & 0 & 0 & 0 \\
\hline$a^{3}$ & 0 & 0 & 0 & 0 \\
\hline$a^{4}$ & 0 & 0 & 0 & $a^{3}$ \\
\hline
\end{tabular}

Figure 1: The modifications of $\mathbf{Z}_{5}$ 
Theorem 8 Let $U \triangleleft S$. Then the sequence

$$
0 \longrightarrow H_{0}^{2}\left(S / U, P^{\times}\right) \stackrel{\chi}{\longrightarrow} H_{0}^{2}\left(S, L^{\times}\right) \stackrel{\psi}{\longrightarrow} H^{2}\left(U, L^{\times}\right)
$$

is exact.

Corollary 9 If the field $L$ is finite then

$$
H_{0}^{2}\left(S, L^{\times}\right) \cong H_{0}^{2}\left(S / U, P^{\times}\right)
$$

Therefore, for finite fields the problem is reduced to the computation of 0 -cohomology of a nilpotent 0 -cancellative semigroup $(S / U) \backslash\{1\}$. I believe that such an algorithm can be built.

At last note that W. Clark [5] used 0-cohomology (however with trivial 0 -modules only) for investigation of some matrix algebras.

\section{Partial cohomologies}

0-Cohomology has one more application: for calculating of EM-cohomology. However from this point of view it is worth once more to generalize our construction.

One can ask: what would be if we considered partial maps as cochains, starting from an arbitrary subset $W \subseteq S$, not necessary from $S \backslash\{0\}$ ? It was shown in [17] that this question is reduced to the following particular case.

Let a semigroup $S$ be generated by a subset $W$ with defining relations of the form $x y=z$ for some $x, y, z \in W$. Such a $W$ will be called a root of $S$. We denote by $W_{n}$ a set of all $n$-tuples $\left(x_{1}, \ldots, x_{n}\right)$ such that $x_{i} x_{i+1} \cdot \ldots \cdot x_{j} \in W$ for all $1 \leq i \leq j \leq n$. Every map from $W_{n}$ to a $S$-module $A$ is called $a$ partial n-dimensional cochain of $W$ or a $W$-cochain with values in $A$. $n$ Dimensional $W$-cochains form an Abelian group $C^{n}(S, W, A)$ for $n>0$. We set $C^{0}(S, W, A)=A$, and if $W_{n}=\emptyset$ then $C^{n}(S, W, A)=0$. The coboundary homomorphism is given by the same formula (1); the corresponding partial cohomology groups (or $W$-cohomology groups) are denoted by $H^{n}(S, W, A)$.

It is clear that we obtain EM-cohomology if $W=S$. Reducing 0cohomology to a partial one looks more complicated: if $S$ is a semigroup with $0, W=S \backslash\{0\}$, then we generate a new semigroup $T=\langle W\rangle$ with the 
operation $*$ and defining relations of the form $u * v=w$, where $u, v, w \in W$ and $u v=w$ in $S$. Then $W$ is a root in $T$ and

$$
H_{0}^{n}(S, A) \cong H^{n}(T, W, A) .
$$

Having a presentation of a semigroup $S$ one can easily build some of its roots.

Example 3 Let $S=\langle a, b, c, d \mid a b=c d\rangle($ see $(2))$. Then

$$
W=\{a, b, c, d, x=a b\}, \quad W_{2}=\{(a, b),(c, d)\}, \quad W_{3}=\emptyset
$$

and

$$
S=\langle a, b, c, d, x \mid a b=x, c d=x\rangle
$$

How are $H^{n}(S, W, A)$ and $H^{n}(S, A)$ connected?

The embedding $W \hookrightarrow S$ induces homomorphisms

$$
\theta_{W}^{n}: H^{n}(S, A) \longrightarrow H^{n}(S, W, A),
$$

Proposition 10 [18] If $W$ is a root of a semigroup $S$ then $\theta_{W}^{n}$ is an isomorphism for $n \leq 1$ and a monomorphism for $n=2$.

Generally speaking, $\theta_{W}^{2}$ can be non-surjective (by the way it means that partial cohomology ought not be a derived functor in the category of $S$ modules).

Proposition 10 enables us to use partial cohomology for calculating 1dimensional EM-cohomology of semigroups (and getting some information about 2-dimensional one) in the case when one succeeds to find a 'good' root in a given semigroup. For instance, consider the semigroup $S$ from Example 3. Define for each $f \in Z^{2}(S, W, A)$ the 1-dimensional $W$-cochain $h$ by

$$
h(s)= \begin{cases}f(a, b), & \text { if } s=a, \\ f(c, d), & \text { if } s=c, \\ 0, & \text { otherwise. }\end{cases}
$$

Then $f=\partial h$, so $H^{2}(S, W, A)=0$. Since $\theta_{W}^{2}$ is injective, $H^{2}(S, A)=0$ too.

To study $\theta^{n}$ for $n>1$ we need some new definitions.

Let $S$ be a semigroup, $W$ be its root. A decomposition $x=x_{1} \ldots x_{k}$ $\left(x_{i} \in W\right)$ of an element $x \in S \backslash W$ is called reduced if $x_{i} x_{i+1} \ldots x_{j} \notin W$ 
for each $i, j, 1 \leq i<j \leq k$. We mean that a reduced decomposition of an element $x \in W$ is its decomposition into the product of one multiplier. A root $W$ is said to be canonic if each element $x \in S$ has the unique reduced decomposition.

For example, the set of all element of $S$ is a canonic root.

A root $W$ is called a $J$-root if $x y=x, y z=z$ implies $x z \in W$ for all $x, y, z \in W$.

Theorem 11 [18, 23] If $W$ is a canonic J-root of $S$, then $\theta_{W}^{n}$ are isomorphisms for all $n \geq 0$.

As above, we can use Theorem 11 for calculating EM-cohomology in higher dimensions. For example, if $S=T * U$ is the free product of semigroups $T$ and $U$, then $W=T \cup U$ is its canonic $J$-root and $W_{n}=T_{n} \cup U_{n}$. Thus, we get

$$
H^{n}(S, A) \cong H^{n}(S, W, A) \cong H^{n}(T, A) \oplus H^{n}(U, A)
$$

for every $S$-module $A$. Below we consider less trivial examples.

Example 4 Let $S=\left\langle a, b_{1}, b_{2}, \ldots \mid a P=Q\right\rangle$ be such a semigroup that the words $P$ and $Q$ do not contain the letter $a$. Denote $W=F \cup\{a\}$, where $F=\left\langle b_{1}, b_{2}, \ldots\right\rangle$ is a subsemigroup of $S$. Then $W$ is a canonic $J$-root. The fact that $F$ is free facilitates the calculation of $W$-cohomology of $S$; so we obtain $H^{2}(S, A)=0$ for every $X$-module $A$ (that is c.d. $S=1$ ).

Example 5 The semigroup

$$
S^{\mathrm{op}}=\left\langle a, b_{1}, b_{2}, \ldots \mid P a=Q\right\rangle,
$$

is antiisomorphic to $S$ (see Example 4). Like for $S$, the subset $W=F \cup\{a\}$ is a canonic $J$-root. However c.d. $S^{\text {op }}=2$. Besides, $H^{2}\left(S^{\mathrm{op}}, A\right) \cong A / B$, where

$$
B=P A+\sum_{i}\left(\frac{\partial P}{\partial b_{i}}-\frac{\partial Q}{\partial b_{i}}\right) A
$$

here $\frac{\partial}{\partial b}$ is an analog of the Fox' derivative [7] adapted to semigroups in [18].

Consider one more pair of antiisomorphic semigroups. 
Example 6 Let $U$ be an arbitrary semigroup,

$$
T=\langle U, p \mid U p=p\rangle \quad(p \notin U)
$$

This notation means that $T$ is generated by its subsemigroup $U$ and by an element $p \notin U$ and is defined by relations of the form

$$
u \cdot v=u v, \quad u \cdot p=p \quad(u, v \in U)
$$

The subset $W=U \cup\{p\}$ turns out a canonic $J$-root. We get c.d. $T=1$ and $H^{1}(T, A) \cong A /(p-1) A$ for every $T$-module $A$.

Example 7 Now consider the semigroup

$$
T^{\mathrm{op}}=\langle U, p \mid p U=p\rangle,
$$

antiisomorphic to $T$. Its EM-cohomology is much more complicated:

Proposition 12 Let $A$ be a $T^{\mathrm{op}}$-module, $A_{1}$ be its additive group considered as a trivial $T^{\mathrm{op}}$-module. The homomorphisms $\psi^{n}: H^{n}\left(T^{\mathrm{op}}, A\right) \rightarrow H^{n}(U, A)$ induced by the embedding $U \hookrightarrow T^{\mathrm{op}}$ are inserted into the long exact sequence

$$
\begin{array}{r}
0 \rightarrow H^{0}\left(T^{\mathrm{op}}, A\right) \stackrel{\psi^{0}}{\rightarrow} H^{0}(U, A) \rightarrow H^{0}\left(U, A_{1}\right) \rightarrow H^{1}\left(T^{\mathrm{op}}, A\right) \stackrel{\psi^{1}}{\rightarrow} \ldots \\
\ldots \rightarrow H^{n}\left(T^{\mathrm{op}}, A\right) \stackrel{\psi^{n}}{\rightarrow} H^{n}(U, A) \rightarrow H^{n}\left(U, A_{1}\right) \rightarrow \ldots
\end{array}
$$

By the last two examples we can build a semigroup $T$ such that c.d. $T=1$ and c.d. $T^{\mathrm{op}}=\infty$. To do it take the additive group of the $\operatorname{ring} \mathbf{Z}_{9}$ as $A$ and its multiplicative group as $U$. The action of $U$ on $A$ coincides with the multiplication in $\mathbf{Z}_{9}$. Then $H^{n}\left(T^{\mathrm{op}}, A\right) \cong \mathbf{Z}_{3}$ for $n>1$.

It is worth to add that the notion of a canonic root can be applied to algorithmic problems. For instance, with its help a new family of semigroups with solvable word problem was obtained [11].

\section{References}

[1] N. Bernstein. On the cohomology of semigroups. Dissert. Abstrs., 25(1965), N1, 6644-6645. 
[2] K. S. Brown. Cohomology of Groups. Springer-Verlag, 1982.

[3] H. Cartan and S. Eilenberg. Homological Algebra. Princeton, 1956.

[4] C. C. Cheng and B. Mitchell. DCC posets of cohomological dimension one. J. Pure and Appl. Algebra, 13(1978), N2, 125-137.

[5] W. E. Clark. Cohomology of semigroups via topology with an application to semigroup algebras. Commun. Algebra, 4(1976), 979-997.

[6] A. H. Clifford and G. B. Preston. The Algebraic Theory of Semigroups. vol.1, 2, AMS Math. Surveys, 1964, 1967.

[7] R. H. Crowell and R.H. Fox. Introduction to Knot Theory. Ginn \& Co, 1967.

[8] C. W. Curtis and I. Reiner. Representation Theory of Finite Groups and Associative Algebras. Willey \& Sons, N.-Y., L., 1967.

[9] D. E. Haile. The Brauer monoid of a field. J. Algebra, 81(1983), N2, 521-539.

[10] D. E. Haile, R. G. Larson and M. E. Sweedler. A new invariant for C over $\mathbf{R}$ : almost invertible cohomology theory and the classification of idempotent cohomology classes and algebras by partially ordered sets with Galois group action. Amer. J. Math., 105(1983), N3, 689-814.

[11] O.S. Kashcheeva and B. V. Novikov. Canonic subsets in semigroups. Filomat (Yugoslavia), 12(1998), N1, 21-27.

[12] V. V. Kirichenko and B. V. Novikov. On the Brauer monoid for finite fields. In: Proc. of 5th Internat. Conf. on Finite Fields and Appl. (to appear).

[13] B. Mitchell. On the dimension of objects and categories. I. Monoids. J. Algebra, 9(1968), N3, 314-340.

[14] B. V. Novikov. On 0-cohomology of semigroups. In: "Theor. and Appl. Quest. of Diff. Equat. and Algebra". Kiev, Naukova dumka, 1978, 185188 (in Russian). 
[15] B. V. Novikov. On projective representations of semigroups. Doklady AN USSR, 1979, N6, 474-478 (in Russian).

[16] B. V. Novikov. An counter-example to a Mitchell conjecture. Trudy Tbiliss. matem. inst. AN GSSR, 70(1982), 52-55 (in Russian).

[17] B. V. Novikov. On partial cohomologies of semigroups. Semigroup Forum, 28(1984), N1-3, 355-364.

[18] B. V. Novikov. Partial cohomologies and their applications. Izv. vuzov. Matem., 1988, N11, 25-32 (in Russian). Translation in Soviet Math. (Iz. VUZ) v.32 (1988), N11, 38-48.

[19] B. V. Novikov. Commutative cancellative semigroups of dimension 1. Matem. Zametki, 48(1990), N1, 148-149 (in Russian).

[20] B. V. Novikov. On the Brauer monoid. Matem. Zametki, 57(1995), N4, 633-636 (in Russian). Translation in Math. Notes 57 (1995), no. 3-4, 440-442.

[21] B. V. Novikov. On modification of the Galois group. Filomat (Yugoslavia), 9(1995), N3, 867-872.

[22] B. V. Novikov. Semigroups of cohomological dimension 1. J. Algebra, 204(1998), 386-393.

[23] B. V. Novikov. Partial cohomologies and canonic roots in semigroups. Matem. studii (Ukraine), 12(1999), N1, 7-14 (in Russian).

[24] B. V. Novikov. On cohomological dimension of ideals of free semigroups. In: Colloq. on Semigroups. July 17-21, 2000. Szeged. Abstracts, 19.

B.V.Novikov, Saltovskoye shosse 258, apt.20, Kharkov, 68178, Ukraine e-mail: boris.v.novikov@univer.kharkov.ua 